\title{
Trombectomia na Angioplastia Primária em Ponte de Safena
}

\author{
Fábio Cardoso de Carvalho', Hugo Alexandre Ross Yokoyama², Armando Elias Chamma ${ }^{3}$, \\ João Henrique Castro33, Maria Clara Pinheiro Manoel'1, Ivone Daroz ${ }^{1}$, Edson Antonio Bregagnollo'
}

\section{RESUMO}

O emprego da trombectomia na angioplastia primária demonstra bons resultados clínicos e angiográficos imediatos, por meio de redução da embolização distal e do fenômeno do no-reflow e otimização da perfusão miocárdica. Essa técnica, principalmente usada em artérias coronárias, em raras oportunidades é descrita quando empregada em pontes de safena aortocoronarianas. Relatamos o caso de um paciente de 67 anos com infarto agudo do miocárdio relacionado a oclusão de ponte de safena e submetido a trombectomia com bons resultados.

DESCRITORES: Angioplastia transluminal percutânea coronária. Veia safena. Trombose coronária. Trombectomia.

\begin{abstract}
Thrombectomy in Primary Angioplasty Related to a Saphenous Graft

Thrombectomy in primary angioplasty has demonstrated good early clinical and angiographic outcomes, preventing distal embolization and the "no-reflow" phenomenon and optimizing myocardial perfusion. This technique is frequently used in native coronary arteries and is rarely reported in coronary grafts. We report the case of a 67-year-old male patient with acute myocardial infarction related to the occlusion of a saphenous vein graft submitted to thrombectomy with good results.
\end{abstract}

DESCRIPTORS: Angioplasty, transluminal, percutaneous coronary. Saphenous vein. Coronary thrombosis. Thrombectomy.

na tentativa de aumentar a segurança e a eficácia do procedimento. Esses sistemas têm se mostrado efetivos na redução da embolização e consequente otimização dos fluxos coronariano e tissular. Há evidências de melhora na evolução clínica intra-hospitalar e a longo prazo ${ }^{2}$. As intervenções coronarianas percutâneas eletivas em enxertos de veia safena trazem consigo incidências aumentadas de embolização distal em comparação às realizadas em vasos nativos, em decorrência da maior carga trombótica da lesão-alvo, associada a piores desfechos clínico-angiográficos imediatos. É infrequente a descrição do uso dos sistemas de trombectomia em enxerto venoso coronariano no contexto da intervenção coronariana percutânea primária e nenhum relato do emprego do cateter Pronto ${ }^{\mathrm{TM}}$ (Vascular Solutions - Minneapolis, Estados Unidos).

\section{RELATO DO CASO}

Paciente do sexo masculino, 67 anos, hipertenso e dislipidêmico, com angina de início recente, coronariografia com lesões multiarteriais e boa função ventricular esquerda. Submetido a revascularização miocárdica cirúrgica um mês após a coronariografia, com

\footnotetext{
${ }^{1}$ Hospital das Clínicas, Faculdade de Medicina de Botucatu, UNESP - Botucatu, SP, Brasil.

${ }^{2}$ Hospital Estadual de Bauru - UNESP - Bauru, SP, Brasil.

${ }^{3}$ Santa Casa de Misericórdia Botucatuense - Botucatu, SP, Brasil. Correspondência: Fábio Cardoso de Carvalho. Rua Florindo Silva, 208 - Jardim Paraíso II - Botucatu, SP, Brasil - CEP 18610-150 E-mail: fabioccarvalho@hotmail.com

Recebido em: 22/9/2008 • Aceito em: 9/1/2009
} 
anastomose da artéria torácica interna esquerda para a descendente anterior (ATIE-DA) e enxertos venosos entre a aorta e as artérias coronárias direita (PS Ao-CD), primeira marginal (PS Ao-Mg) e primeira diagonal esquerda (PS Ao-Dg), persistiu assintomático nos três meses subsequentes à cirurgia, quando precisou ser reinternado com dor precordial típica ao repouso há 40 minutos. À admissão, o exame físico demonstrou estabilidade hemodinâmica e o eletrocardiograma evidenciou supradesnivelamento do segmento ST nas derivações D1 e aVL (Figura 1A). Após ser medicado com 600 mg de clopidogrel, 200 mg de ácido acetilsalicílico (AAS), 5 mg de metoprolol endovenoso e analgesia, o paciente foi encaminhado à sala de hemodinâmica.

A cinecoronariografia evidenciou artéria coronária direita com lesão grave proximal e oclusão do enxerto PS Ao-CD, artéria descendente anterior com lesão grave proximal e anastomose da ATIE-DA normofuncionante, artéria circunflexa com lesão grave proximal e PS Ao-Mg normofuncionante, primeira diagonal ocluída no terço proximal e enxerto PS Ao-Dg ocluído na anastomose distal, com grande carga de trombo local e retenção de contraste no leito do enxerto venoso (Figuras 2A e 2B).

O manejo farmacológico em sala consistiu inicialmente na administração de $60 \mathrm{UI} / \mathrm{kg}$ de heparina nãofracionada e inibidor de glicoproteína Ilb/Illa (tirofiban), posicionamento de cateter-guia AL1 6 Fr e corda-guia hidrofílica até o leito distal do primeiro ramo diagonal. O cateter Pronto ${ }^{\mathrm{TM}}$ (Vascular Solutions - Minneapolis, Estados Unidos) foi posicionado junto à extremidade proximal do trombo e procedeu-se à trombectomia com seringa a vácuo. Após retirado o cateter de trombectomia, foi observada extração de pequeno fragmento de trombo (Figura 2C); o fluxo coronariano no leito distal do vaso nativo reconstituiu-se fluxo TIMI 1, a despeito da permanência de grande quantidade de trombo. Após nova manobra de trombectomia, observou-se oclusão do cateter-guia. Todo o sistema de angioplastia foi retirado e, após lavagem da luz do cateter-guia, foram obtidos dois grandes fragmentos de trombo (Figura 2C). Nova angiografia do enxerto evidenciou fluxo coronariano TIMI 2-3.

A seguir, foram administrados $500 \mu \mathrm{g}$ de verapamil e $10 \mathrm{mg}$ de mononitrato pelo cateter-guia. Depois de alguns minutos, novo controle angiográfico evidenciou fluxo coronariano e tissular 3 pelo enxerto e leito distal da primeira diagonal (Figura 3).

Houve expressivo alívio sintomático e diminuição acentuada do supradesnivelamento do segmento ST no monitor cardíaco e no eletrocardiograma pós-procedimento (Figura 1B). Na angiografia final, não foi observada lesão na anastomose distal do enxerto venoso ou no leito nativo da artéria coronária.

Pós-angioplastia, houve melhora eletrocardiográfica (redução de mais de $50 \%$ do supradesnivelamento do segmento ST nas derivações D1 e aVL, com persistência de onda R) e clínica, com ausência de sintomas durante a internação. O tratamento farmacológico adjunto consistiu de clopidogrel $75 \mathrm{mg} / \mathrm{dia}$, AAS $200 \mathrm{mg} / \mathrm{dia}$, metoprolol $100 \mathrm{mg} / \mathrm{dia}$, enalapril $10 \mathrm{mg} / \mathrm{dia}$, e sinvastatina $40 \mathrm{mg} /$ dia. A alta hospitalar ocorreu no quinto dia pós-procedimento.

\section{DISCUSSÃO}

A presença de carga trombótica em ACP é fator limitante na obtenção de fluxos epicárdico e tissular 3 após o procedimento. Há evidências de que a grande carga trombótica, além de limitar o sucesso do procedimento, limita também seu sucesso clínico ${ }^{1,2}$. A embolização distal, presente em cerca de 15\% dos casos, associa-se a pior perfusão miocárdica, maior extensão do dano miocárdico e prognóstico pior, com risco relativo de 8,6 em cinco anos de seguimento ${ }^{1}$. Alguns relatos demonstraram a eficácia e a segurança dos dispositivos de aspiração de trombo durante a ACP, particularmente quando há grande quantidade de trombo visível à
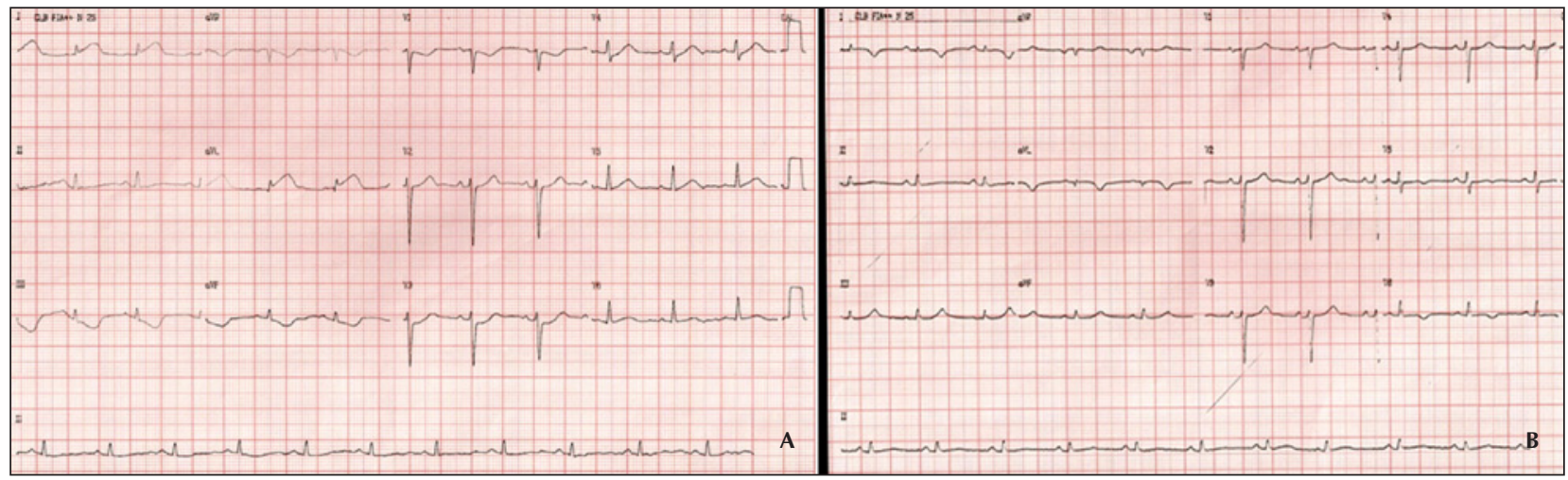

Figura 1 - Em A, eletrocardiograma realizado à admissão do paciente no pronto-socorro, evidenciando supradesnivelamento do segmento ST nas derivações laterais altas (D1 e aVL). Em B, eletrocardiograma pós-procedimento, evidenciando resolução completa do supradesnivelamento do segmento ST. 

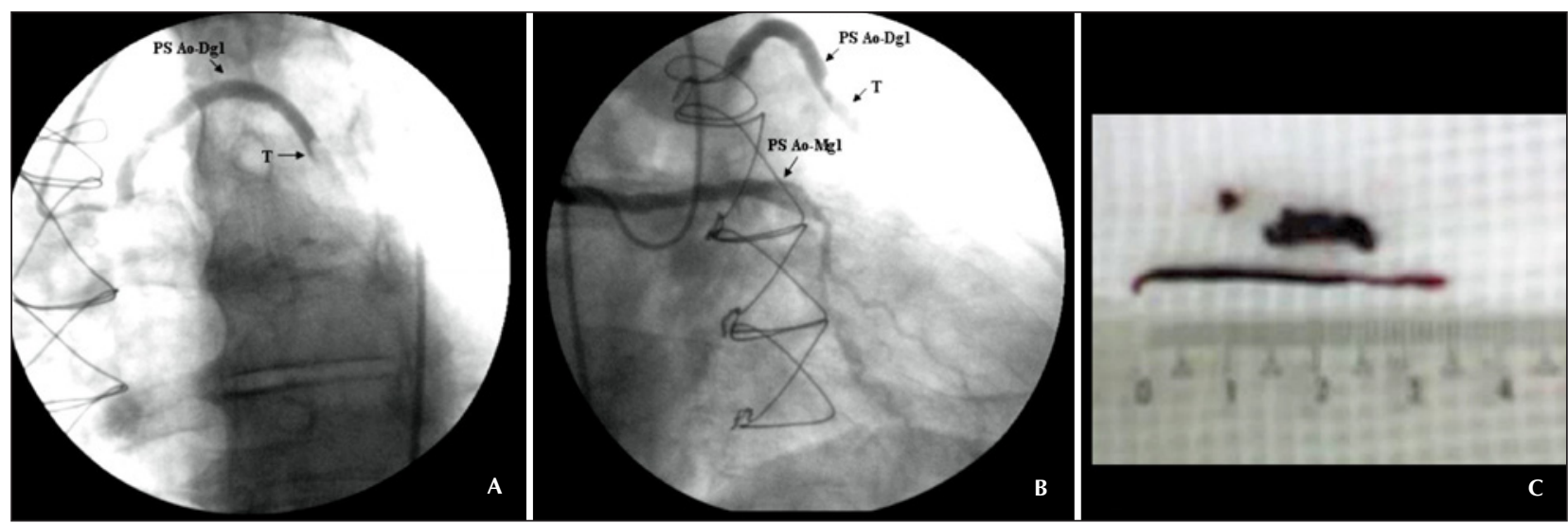

Figura 2 - Em A, angiografia no enxerto ponte de safena aorta-primeira diagonal (PS Ao-Dg1), evidenciando grande carga de trombo na anastomose distal (T). Em B, angiografia no enxerto venoso entre a aorta e a primeira marginal (PS Ao-Mg1), evidenciando a retenção de contraste no enxerto PS Ao-Dg1, com grande carga de trombo em T. Em C, fotografia dos três fragmentos de trombo retirados do enxerto venoso coronariano, utilizando o cateter Pronto $^{\mathrm{TM}}$.

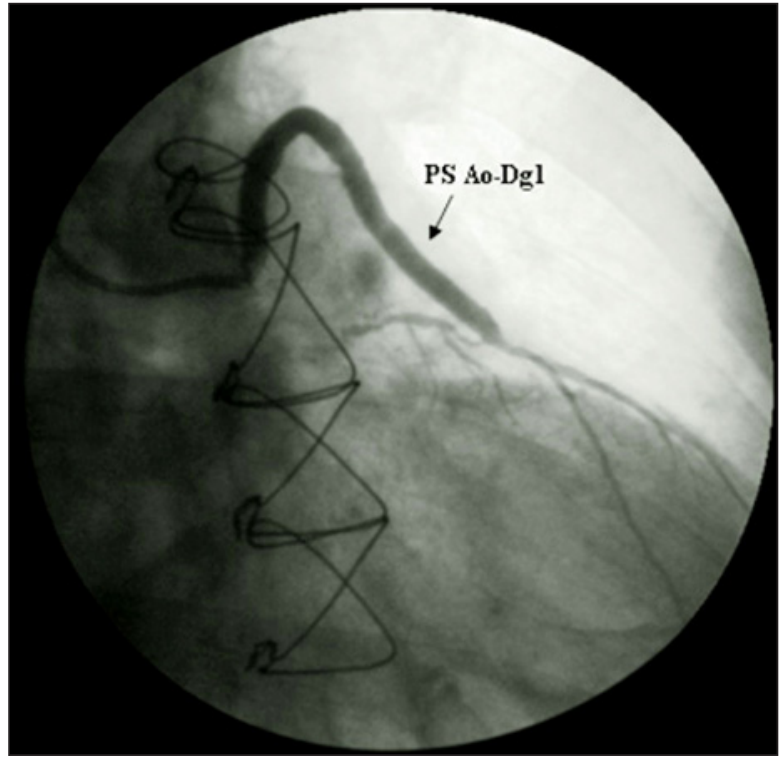

Figura 3 - Angiografia no enxerto coronariano ponte de safena aorta-primeira diagonal (PS Ao-Dg1), evidenciando fluxo coronariano normal através do enxerto (TIMI 3, blush miocárdico 3).

angiografia ${ }^{3-6}$, com melhores resultados angiográficos imediatos, porém sem benefício consistente a longo prazo. Young et al. ${ }^{7}$, no registro X-SIZER for TReatment of thrombus and Atherosclerosis in Coronary interventions Trial in Acute Myocardial Infarction (X-TRACT AMI), demonstraram que a trombectomia antes do implante do stent, no infarto agudo do miocárdio, tanto em artérias coronárias nativas como em pontes de safena degeneradas $(10 \%$ dos casos nesse registro), melhorou o resultado angiográfico do procedimento, tendo alcançado sucesso clínico em $80 \%$ dos pacientes, mesmo com uso de inibidores de glicoproteína IIb/IIla (86\% dos casos).
Os estudos randomizados que avaliaram esses dispositivos (filtros de proteção distal, cateteres de trombectomia) na tentativa de redução da incidência da embolização distal também encontraram resultados conflitantes. Taguchi et al. ${ }^{8}$ relataram a superioridade no resultado angiográfico imediato do sistema de proteção distal sobre a aspiração de trombo na ACP (fluxo TIMI 3 em 94,4\% no grupo proteção vs. $79 \%$ no grupo aspiração, $\mathrm{P}<0,01$ e blush miocárdico 3 em $56,6 \%$ no grupo proteção vs. $33,3 \%$ no grupo aspiração, $\mathrm{P}<0,01)$. O estudo Enhanced Myocardial Efficacy and Recovery by Aspiration of Liberated Debris $\left(\right.$ EMERALD) ${ }^{9}$ avaliou 501 pacientes randomizados para proteção distal ou não na $\mathrm{ACP}$, não tendo encontrado diferença significante com respeito a fluxo distal (TIMI), redução do tamanho do infarto ou sobrevida livre de eventos adversos relacionados a disfunção sistólica do ventrículo esquerdo ou complicações isquêmicas do infarto no período de até seis meses, nas duas estratégias de abordagem. O estudo prospectivo Randomized Evaluation of the Effect of Mechanical Reduction of Distal Embolization by Thrombus-Aspiration in Primary and Rescue Angioplasty (REMEDIA) ${ }^{10}$ avaliou os resultados angiográficos imediatos na ACP de 100 pacientes consecutivos ( $\mathrm{n}=50$ no grupo aspiração e $n=49$ no grupo convencional), tendo relatado meIhores resultados imediatos do procedimento no grupo aspiração (fluxo TIMI 3 e resolução do segmento ST), e ausência de diferença na incidência de eventos cardíacos maiores (morte, reinfarto, acidente vascular encefálico, revascularização da lesão-alvo) nos pacientes dos dois grupos em 30 dias. Resultados semeIhantes foram encontrados por Silva-Orrego et al. ${ }^{11}$, no estudo Dethrombosis to Enhance Acute Reperfusion in Myocardial Infarction (DEAR-MI), avaliando os mesmos desfechos, no mesmo período. Uma importante metanálise sobre o assunto foi publicada em 2007, num total de 21 estudos, com 3.721 pacientes $^{12}$. Os 
achados desse estudo reforçaram o benefício dos sistemas de prevenção da embolização distal nos resultados imediatos da ACP (fluxo TIMI 3, blush miocárdico 3 e menor embolização distal), mas sem haver benefício clínico, como diminuição da mortalidade em 30 dias. Estudos mais recentes ${ }^{2,13,14}$ demonstram que os resultados imediatos alcançados com a utilização desses sistemas têm sido acompanhados de diminuição da mortalidade em até um ano. Javaid et al. $^{13}$ demonstraram, em 158 pacientes, que no grupo no qual o trombo foi aspirado $(n=80)$, comparado ao grupo da ACP convencional $(\mathrm{n}=78)$, não só $\mathrm{O}$ resultado angiográfico foi melhor (fluxo TIMI $3 \mathrm{em}$ $91,3 \%$ vs. $67,9 \%$; $\mathrm{P}<0,001)$ como os eventos cardíacos maiores (morte e infarto agudo do miocárdio não-fatal) e a mortalidade em seis meses $(5,4 \%$ vs. $21,3 \% ; P=0,009)$ e em um ano $(7,7 \%$ vs. $26,2 \%$; $\mathrm{P}=0,005)$ foram significantemente menores. Nesse estudo, 5\% dos pacientes do grupo aspiração de trombo e $8 \%$ do grupo ACP convencional eram portadores de enxertos de veia safena. O Thrombus Aspiration during Percutaneous coronary intervention in Acute myocardial infarction Study (TAPAS) ${ }^{14}$ demonstrou resultados semelhantes em 30 dias. Nesse estudo, os 535 pacientes do grupo aspiração de trombo, comparados aos 536 pacientes do grupo ACP convencional, obtiveram melhor fluxo TIMI, melhor blush miocárdico e resolução do segmento ST, resultando em menor incidência de eventos cardíacos adversos maiores (morte, reinfarto ou revascularização da lesão-alvo), com taxas de 14,1\% para blush 0 , de $8,8 \%$ para blush 1 e 2 , e de $4,2 \%$ para blush $3(\mathrm{P}=0,003)$ e menor mortalidade $(5,2 \%$ para blush 0, 2,9\% para blush 1 e 2, e $1 \%$ para blush 3; $\mathrm{P}<0,001)$. O inibidor de glicoproteína IIb/IIla foi utilizado em 93,4\% dos casos do grupo aspiração e em $89,9 \%$ do grupo ACP convencional ( $>>0,05)$. A análise de um ano desses mesmos grupos de pacientes confirmou a manutenção do benefício nesse período, com taxas de mortalidade de $3,6 \%$ no grupo aspiração do trombo e de $6,7 \%$ no grupo ACP convencional $(P=0,02)$ e ocorrência de morte ou infarto não-fatal em $5,6 \%$ dos pacientes do grupo aspiração e em 9,9\% do grupo ACP convencional $(P=0,009)$.

\section{CONCLUSÃO}

No caso descrito, a utilização do cateter extrator de trombo Pronto ${ }^{\mathrm{TM}}$ na ACP mostrou-se fácil, segura e mesmo imprescindível para o sucesso do procedimento. Essa ferramenta tem se mostrado útil nos procedimentos na fase aguda do infarto agudo do miocárdio, com evidência de benefício imediato e desfechos clínicos tardios. Seu uso universal ainda não é recomendado, mas já é contemplado nas diretrizes de intervenção coronária percutânea da Sociedade Brasileira de Cardiologia e Sociedade Brasileira de Hemodinâmica e Cardiologia Intervencionista ${ }^{15}$, como classe lla (nível de evidência B), na ACP.

\section{CONFLITO DE INTERESSES}

Os autores declararam inexistência de conflito de interesses.

\section{REFERÊNCIAS BIBLIOGRÁFICAS}

1. Henriques JP, Zijlstra F, Ottervanger JP, de Boer MJ, van't Hof AW, Hoorntje JC, et al. Incidence and clinical significance of distal embolization during primary angioplasty for acute myocardial infarction. Eur Heart J. 2002;23(14):1112-7.

2. Svilaas T, Vlaar PJ, van der Horst IC, Diercks GF, de Smet BJ, van den Heuvel AF, et al. Thrombus aspiration during primary percutaneous coronary intervention. $N$ Engl J Med. 2008;358(6):557-67.

3. Topaz O, Perin EC, Jesse RL, Mohanty PK, Carr M Jr, Rosenschein $U$. Power thrombectomy in acute ischemic coronary syndromes. Angiology. 2003;54(4):457-68.

4. Lim MJ, Reis L, Ziaee A, Kern MJ. Use of a new thrombus extraction catheter (the Pronto) in the treatment of acute myocardial infarction. J Interv Cardiol. 2005;18(3):189-92.

5. Pershad A, Hoelzinger D, Patel S. Pronto catheter thrombectomy in acute ST-segment myocardial infarction: a case series. J Invasive Cardiol. 2006;18(6):E191-4.

6. Santos MA, Fonseca CGB, Godoy MF. Eficácia e segurança do cateter "Pronto" para aspiração manual de trombo durante angioplastia primária no infarto agudo do miocárdio com elevação do segmento ST. Rev Bras Cardiol Invas. 2007;15(2):141-4.

7. Young JJ, Cox DA, Stuckey T, Babb J, Turco M, Lansky AJ, et al. Prospective, multicenter study of thrombectomy in patients with acute myocardial infarction: The X-Tract AMI registry. J Interv Cardiol. 2007;20(1):44-50.

8. Taguchi I, Kanaya T, Toi T, Abe S, Sugimura H, Hoshi T, et al. Comparison of the effects of a distal embolic protection device and an aspiration catheter during percutaneous coronary intervention in patients with acute myocardial infarction. Circ J. 2005;69(1):49-54.

9. Stone GW, Webb J, Cox DA, Brodie BR, Qureshi M, Kalynych $A$, et al. Distal microcirculatory protection during percutaneous coronary intervention in acute ST-segment elevation myocardial infarction: a randomized controlled trial. Enhanced Myocardial Efficacy and Recovery by Aspiration of Liberated Debris (EMERALD) Investigators. JAMA. 2005;293(9):1063-72.

10. Burzotta F, Trani C, Romagnoli E, Mazzari MA, Rebuzzi AG, De Vita $M$, et al. Manual thrombus-aspiration improves myocardial reperfusion: The Randomized Evaluation of the Effect of Mechanical Reduction of Distal Embolization by Thrombus-Aspiration in Primary and Rescue Angioplasty (REMEDIA) trial. J Am Coll Cardiol. 2005;46(2):371-6.

11. Silva-Orrego P, Colombo P, Bigi R, Gregori D, Delgado A, Salvade $\mathrm{P}$, et al. Thrombus aspiration before primary angioplasty improves myocardial reperfusion in acute myocardial infarction: the DEAR-MI (Dethrombosis to Enhance Acute Reperfusion in Myocardial Infarction) study. J Am Coll Cardiol. 2006;48(8):1552-9.

12. De Luca G, Suryapranata H, Stone GW, Antoniucci D, Neumann FJ, Chiariello M. Adjunctive mechanical devices to prevent distal embolization in patients undergoing mechanical revascularization for acute myocardial infarction: a meta-analysis of randomized trials. Am Heart J. 2007;153(3):343-53.

13. Javaid A, Siddiqi NH, Steinberg DH, Buch AN, Slottow TL, Roy $\mathrm{P}$, et al. Adjunct thrombus aspiration reduces mortality in patients undergoing percutaneous coronary intervention for ST-elevation myocardial infarction with high-risk angiographic characteristics. Am J Cardiol. 2008;101(4):452-6. 
14. Vlaar PJ, Svilaas T, van der Horst IC, Diercks GF, Fokkema $\mathrm{ML}$, de Smet BJ, et al. Cardiac death and reinfarction after 1 year in the Thrombus Aspiration during Percutaneous coronary intervention in Acute myocardial infarction Study (TAPAS): a 1-year follow-up study. Lancet. 2008;371(9628): 1915-20.
15. Mattos LA, Lemos Neto PA, Rassi A Jr, Marin-Neto JA, Sousa AGMR, Devito FS, et al. Diretrizes da Sociedade Brasileira de Cardiologia - Intervenção Coronária Percutânea e Métodos Adjuntos Diagnósticos em Cardiologia Intervencionista (II Edição - 2008). Rev Bras Cardiol Invas. 2008; 16(Supl. 2):9-88. 